\title{
A Comparison of Cross Section Formulas and their Effect on Calculated k-factors
}

\author{
Alan Sandborg ${ }^{1}$, Patrick Camus ${ }^{2}$, Brent Hammell ${ }^{2}$ \\ ${ }^{1}$ Consultant, 2055 Frost Road, Schaumburg, IL 60195 \\ ${ }^{2}$ EDAX Inc., 91 McKee Drive, Mahwah, NJ 07430
}

In order to calculate Cliff-Lorimer k-factors, the ionization cross section must be incorporated. The ionization cross section has only been measured for a relatively small number of elements and electron energies, so formulas must be used. Powell studied the available data and concluded the Bethe equation could be used with effective values of the Bethe parameters $b_{n l}$ and $c_{n l}$. Many other workers determined different values or fits vs atomic number for these two parameters. One of those workers was Zaluzec, who developed fits to atomic number and use them in his quantitative program for thin section samples called NEDQNT. EDAX has used that formulation for years in the programs $\mathrm{mDX}$ and MTHIN. Sheridan measured many k-factors on a $200 \mathrm{Kv}$ TEM and a SiLi detector with a Be window. He compared the Bethe formula in various forms with different values for $b_{n l}$ and $c_{n l}$. This did not allow the measurement of elements with $Z$ less than 11. Modern SiLi and SDD EDS x-ray detectors can measure elements with $\mathrm{Z}$ as low as 5 , so his data does not allow evaluation of the models for low $\mathrm{Z}$ elements.

In an effort to improve the accuracy of thin section sample analysis on TEM instruments, other methods have been compared to Powell and Zaluzec. Casnati approached the ionization cross section with an empirical formula which is not based on the Bethe formula. Campos used a parameterized model of the distorted-wave first order Born approximation (DWBA). It was decided to compare the Zaluzec, Casnati and Campos cross sections.

Figure 1 shows the calculated k-factors from the 3 models compared to measurements made by Sheridan. The k-factors were calculated for a Be window SiLi detector which was available to Sheridan. The uncertainty in the knowledge of the Be thickness and the nature of the Si dead layer on the SiLi detector may account for the k-factors for $\mathrm{S}, \mathrm{K}$, and $\mathrm{Cl}$. Otherwise the Zaluzec and Casnati models best fit the data. Table 1 shows the average difference for the calculations vs the measured values. Figure 2 shows the calculated k-factors for a windowless SDD compared to data acquired at $200 \mathrm{Kv}$ with the NIST 2063a sample. The experimental data fits best the Zaluzec and Casnati models.

The k-factors calculated using the Zaluzec and Casnati ionization cross section formulas show the best fits to the limited experimental data. While the average errors in quantitative analysis using these calculations approach $5 \%$, for some elements the errors are $10 \%$ or higher.

\section{References:}

[1] C. J. Powell, Reviews of Modern Physics, Vol. 48, No.1, January 1976

[2] N. J. Zaluzec, Analytical Electron Microscopy, 1984, p. 279

[3] N. J. Zalusec, NEDQNT, http://tpm.amc.anl.gov/NJZTools/

[4] P. Sheridan, Journal of Electron Microscopy Technique, 11:41-61 (1989)

[5] E. Casnati, A, Tartari, and C. Baraldi, J. Phys. B: At. Mo;. Phys., 15 (1982) 155-167

[6] C. S. Campos, M. A. Z. Vasconcellos, J. C. Trincavelli and S. Segui, J. Phys. B: At. Mol. Opt. Phys. 40 (2007) 3835-3841 


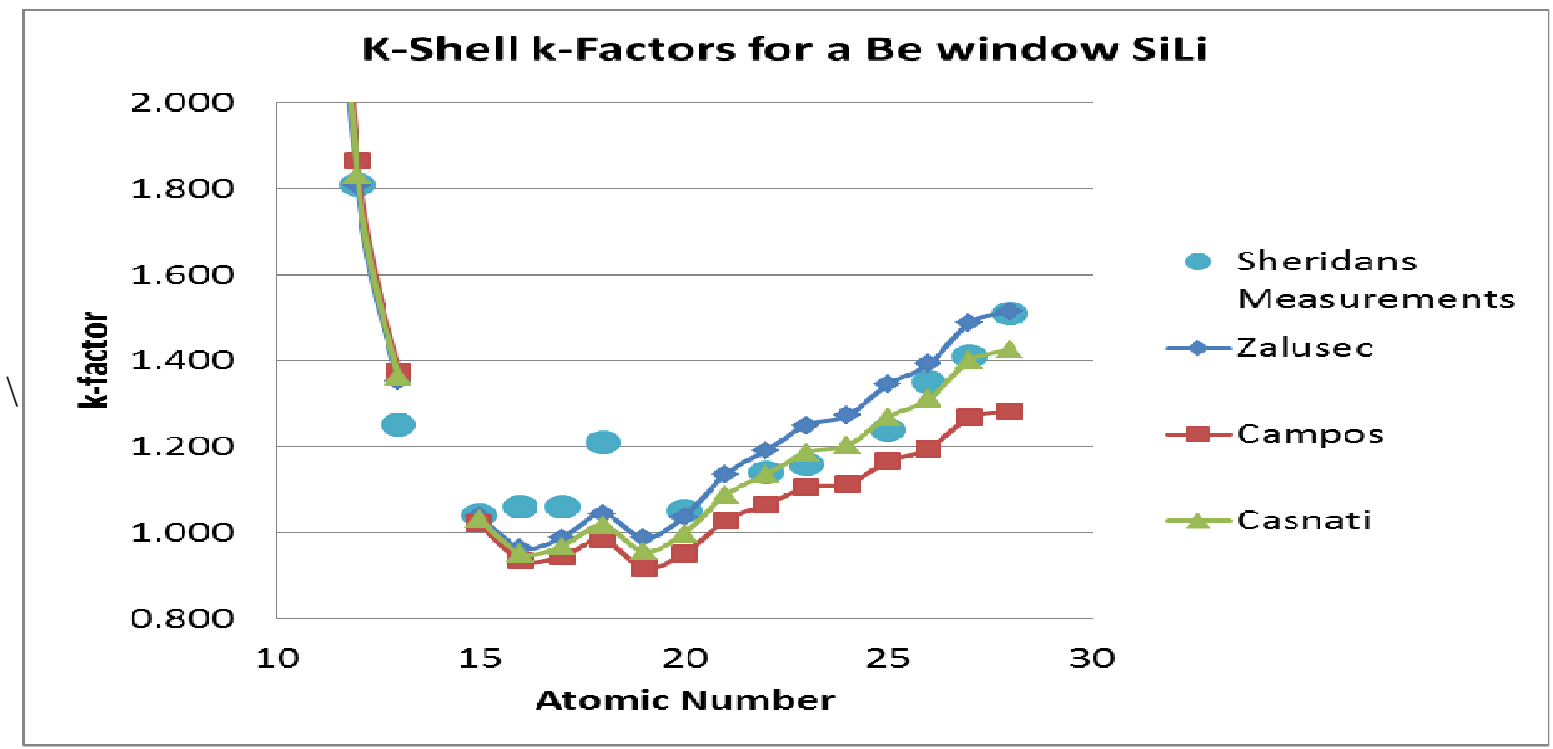

Figure 1. Calculated k-factors vs Sheridan's measured k-factors

\begin{tabular}{|l|l|l|}
\hline Zalusec & Campos & Casnati \\
\hline 5.3 & 9.2 & 5.0 \\
\hline
\end{tabular}

Table 1. Average Percent difference between calculated and Sheridan's measured k-factors.

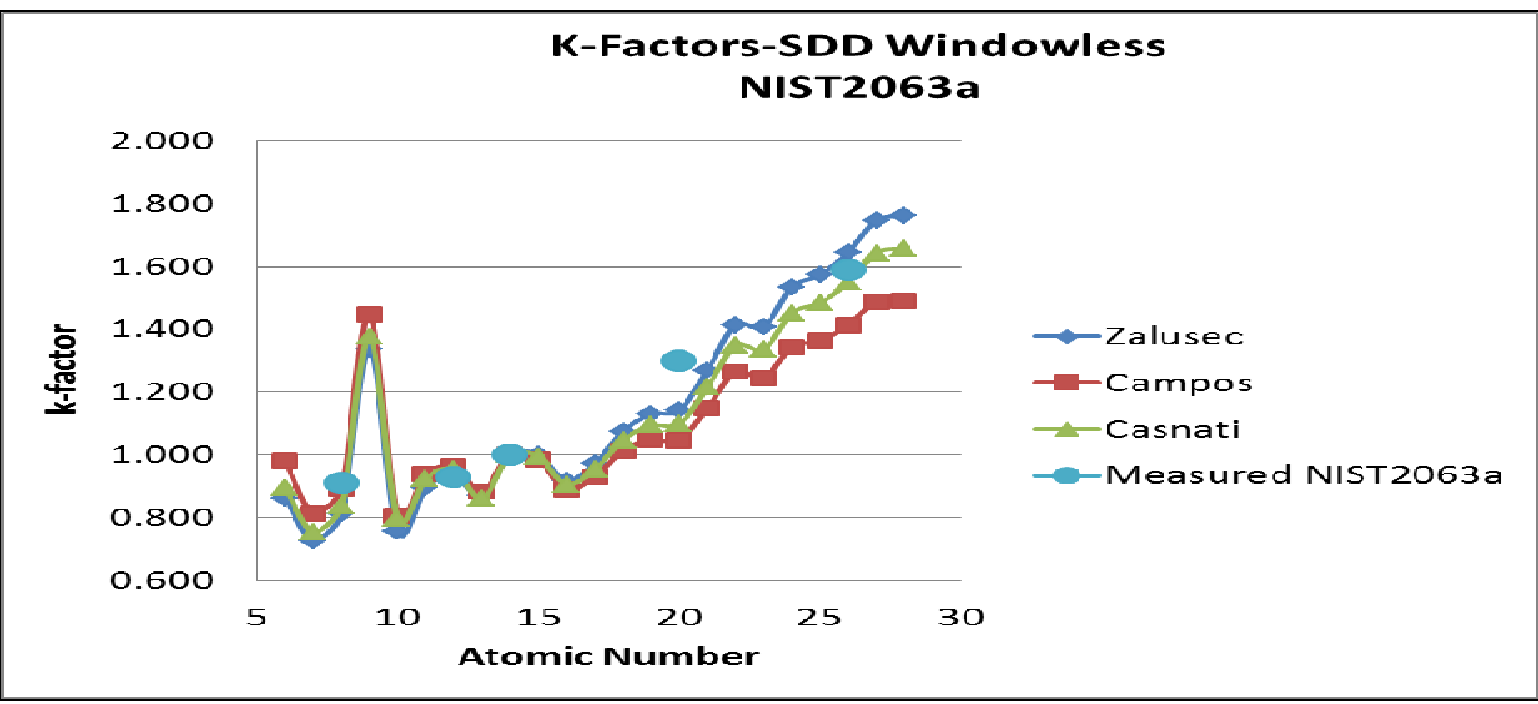

Figure 2. Calculated k-factors for a SDD windowless detector vs measured values for NIST 2063a 